\title{
Perfil sociodemográfico e clínico de crianças com estomia atendidas em um serviço de referência, Belém (PA)*
}

\author{
Sociodemographic and clinical profile of stoma children \\ attending in a reference center, Belém, state of Pará, Brazil
}

\section{Perfil sociodemográfico y clínico de los niños con ostomía atendidos en servicio de referencia, Belém (PA), Brasil}

\author{
Pérola Dias Bezerra', Izabela do Socorro Martins Pinto², Regina Ribeiro Cunha ${ }^{3}$, \\ Edson Marcos Leal Soares Ramos', Cassilene Oliveira da Silva ${ }^{5}$, Sandra Regina Monteiro Ferreira ${ }^{6}$
}

\begin{abstract}
RESUMO
Este estudo teve como objetivo caracterizar o perfil sociodemográfico e clínico de crianças com estomia atendidas em um serviço de referência no estado do Pará, Brasil. Trata-se de um estudo retrospectivo de análise documental, transversal e descritiva, com abordagem quantitativa. Os dados foram coletados em outubro de 2013, analisados e apresentados em frequência absoluta e relativa. Identificaram-se 96 prontuários de crianças com estomia. A maior frequência de crianças com estomia é do sexo masculino, compreendida na faixa etária de 0 a 3 anos, sem escolaridade, pois não está em idade escolar, residente no interior do estado do Pará, cujos pais possuem renda familiar entre um e três salários-mínimos. Com relação às características clínicas, foi evidenciada a malformação congênita como a causa geradora de estomia de maior prevalência. A maioria das crianças apresentava colostomia, terminal ou duas bocas próximas, temporária, localizada no quadrante inferior esquerdo, com diâmetro entre 20 e 29 mm. O prolapso e a dermatite foram as principais complicações relacionadas à estomia e pele peristoma.
\end{abstract}

DESCRITORES: Criança. Estomia. Perfil de Saúde. Estomaterapia.

\section{ABSTRACT}

This study aimed to characterize the sociodemographic and clinical profile of stoma children attending in a reference center in Pará state, Brazil. This is a retrospective study of documental, transverse and descriptive analysis, with a quantitative approach. Data were collected in October 2013, analyzed and presented as absolute and relative frequency. We identified 96 medical records of ostomy children. The highest frequency of ostomy children is male, aged 0 to

\footnotetext{
*Extraído do trabalho de conclusão de curso "Perfil sociodemográfico e clínico de crianças com estomia no contexto amazônico", apresentado à Faculdade de Enfermagem, Instituto de Ciências da Saúde, Universidade Federal do Pará (FAENF/ICS/UFPA), em 2013.

${ }^{1}$ Enfermeira. Coordenadora em Doenças Crônicas e Tabagismo. Membro do Grupo de Pesquisa Enfermagem em Estomaterapia da Amazônia (ENFESTA) - Castanhal (PA), Brasil.

²Enfermeira. Pós-Graduada em Estética Dermaticista pela Faculdade Mario Schenberg/Instituto Brasileiro de Eletroterapia e Cosmetologia de São Paulo. Membro do Grupo de Pesquisa ENFESTA - Belém (PA), Brasil.

"Enfermeira Estomaterapeuta Ti SOBEST. Doutora em Enfermagem. Professora adjunta da Escola de Enfermagem "Magalhães Barata" da Universidade do Estado do Pará (UEPA) e da Faculdade de Enfermagem da Universidade Federal do Pará (UFPA). Membro do Grupo de Pesquisa Intervenções de Enfermagem no Processo Saúde Doença (IENPSAD) e do Grupo de Pesquisa em Estomaterapia: Estomas, Feridas Agudas e Crônicas e Incontinências Urinária e Anal (EEUSP). Líder do Grupo de Pesquisa ENFESTA - Belém (PA), Brasil. Endereço para correspondência: Rua Caripunas, 2.742, apto. 701 CEP: 66045-140 - Belém (PA), Brasil. E-mail: reginaribeirocunha@gmail.com

${ }^{4}$ Estatístico. Doutor em Engenharia de Produção. Professor associado da Faculdade de Estatística da UFPA - Belém (PA), Brasil.

5Enfermeira Graduada pela Escola de Enfermagem "Magalhães Barata" da UEPA. Membro do Grupo de Pesquisa ENFESTA e do Grupo de Pesquisa IENPSAD - Belém (PA), Brasil.

${ }^{6}$ Enfermeira da Fundação Hospital de Clínicas Gaspar Vianna. Mestranda do Programa de Pós-Graduação em Saúde, Ambiente e Sociedade na Amazônia da UFPA. Coordenadora do Serviço de Atenção à Pessoa com Estomia da Secretaria de Estado de Saúde Pública do Pará. Membro do Grupo de Pesquisa ENFESTA - Belém (PA), Brasil.

Artigo recebido em 03/05/2016 - Aceito para publicação em: 09/11/2016
} 
3 years old, without schooling, because they are not at school age, resident in the interior of Pará state and family income between one and three minimum wage. Regarding the clinical characteristics, congenital malformation was evidenced as a generator of the highest prevalence ostomy. Most of the children had temporarily terminal ileostomy, located in the lower left quadrant, with a diameter from 20 to $29 \mathrm{~mm}$. Prolapse and dermatitis were the main complications related to ostomy and periestoma skin.

DESCRIPTORS: Child. Ostomy. Health Profile. Stomatherapy.

\section{RESUMEN}

Este estudio tuvo como objetivo caracterizar el perfil sociodemográfico y clínico de niños con ostomía atendido en servicio de referencia en el estado de Pará, Brasil. Se trata de un estudio retrospectivo de análisis documental, transversal y descriptivo, con enfoque cuantitativo. Los datos fueron recolectados en octubre de 2013, analizados y presentados como frecuencia absoluta y relativa. La muestra consistió en 96 prontuarios de niños con ostomía registrada en dicho servicio. Los datos se recogieron en octubre de 2013.. La mayor frecuencia de los niños con ostomía es masculina, comprendida en el rango de edad de 0 a 3 años, sin escolaridad porque no están en edad escolar, residen en el interior del estado de Pará, el ingreso familiar entre 1 y 3 salarios mínimos. En cuanto a las características clínicas evidenciaron que malformación congénita se constituye como un generador debido a la mayor prevalencia de la ostomía> La mayoría de los niños tenían colostomía, o dos bocas cercanas, temporaria, situada temporalmente, en el cuadrante inferior izquierdo, siendo el diámetro de 20 a 29 mm, prolapso y dermatitis fueron las principales complicaciones relacionadas con la ostomía y la piel periestomia.

DESCRIPTORES: Niño. Ostomía. Perfil de Salud. Estomaterapia.

\section{INTRODUÇÃO}

A ocorrência da doença de Hirschsprung, da malformação anorrectal, da enterocolite necrotizante e ileomeconial, de malformações da coluna lombro sacra e da mielomeningocele constituem algumas indicações para realização de cirurgia geradora de estomia em crianças, na maioria das vezes de caráter temporário, por se tratar de causas benignas ${ }^{1}$. O Estatuto da Criança e do Adolescente (ECA) estabelece que criança é a pessoa compreendida na faixa etária de 0 a 12 anos de idade incompletos ${ }^{2}$. As crianças com estomia são reconhecidas legalmente como pessoas com deficiência física ${ }^{3,4}$.

Entre as estomias digestivas de maior incidência na infância e no período neonatal, destacam-se as colostomias de caráter temporário, que consistem na exteriorização do colón por meio da parede abdominal, cujo objetivo é descomprimir, proteger anastomoses ou restaurar a função de um órgão afetado ${ }^{5}$.

As crianças com estomia apresentam necessidades e cuidados específicos nas diferentes fases de seu crescimento e desenvolvimento, podendo ser biológicos, emocionais, sociais e culturais. Nesse contexto, a assistência prestada pela equipe multiprofissional, especialmente pelo enfermeiro estomaterapeuta, deve estar centrada no processo de adaptação e reabilitação ${ }^{6}$ com foco no desenvolvimento de estratégias para o cuidado integral da criança com os pais/responsáveis, informando-os sobre os recursos da comunidade ${ }^{7,8}$.

No Brasil, alguns estudos têm sido realizados em serviços especializados ${ }^{8,9}$, porém ainda não retratam o perfil nacional das pessoas com estomia. É fundamental disponibilizar serviços com base nas necessidades e demandas dos usuários, considerando dados epidemiológicos da população conforme a Lei n. $.^{\circ} 8.080 / 90^{10}$.

A inexistência de dados acerca do perfil das crianças com estomia pode interferir negativamente no planejamento das ações em saúde, na previsão e na provisão de insumos e na assistência prestada pelos profissionais.

Acreditamos que o estudo seja relevante por contribuir com o planejamento de ações integrais à saúde dessa população. A partir do momento em que se tem acesso ao perfil sociodemográfico e clínico das crianças com estomia, as ações de atenção à saúde a essa população poderão ser planejadas conforme as suas necessidades. Além disso, a presente investigação colaborará com o gerenciamento de cuidados e evidenciará as morbidades relacionadas à confecção de estomia. 


\section{OBJETIVO}

Caracterizar o perfil sociodemográfico e clínico de crianças com estomia atendidas em um serviço de referência no estado do Pará, Brasil.

\section{MÉTODO}

Trata-se de um estudo retrospectivo de análise documental, transversal e descritiva, com abordagem quantitativa, realizado em um serviço de referência em atenção às pessoas com estomia, localizado na cidade de Belém, estado do Pará, Brasil. Teve como critérios de seleção e inclusão prontuários de crianças de ambos os sexos, na faixa etária entre $0 \mathrm{e}$ 12 anos incompletos, cadastradas no período entre outubro de 2009 e outubro de 2012. Identificamos 96 prontuários de crianças com estomia.

Os dados foram coletados em outubro de 2013 e inseridos em instrumento de coleta de dados adaptado ${ }^{11}$. Utilizamos as variáveis sociodemográficas - procedência, sexo, idade, escolaridade e renda familiar - e as variáveis clínicas: causa básica da estomia, tipo de estomia, caráter da estomia, diâmetro da estomia, localização da estomia, modo de exteriorização, complicações relacionadas à estomia e pele periestoma.

Os dados foram organizados em um banco de dados criado no programa Microsoft Excel ${ }^{\circledR}$. A análise dos dados foi realizada por meio da estatística descritiva, e discutiram-se os resultados com base na literatura associada ao tema de estudo.

A investigação obteve aprovação do Comitê de Ética em Pesquisa com Seres Humanos (CEPSH) da Universidade Federal do Pará (UFPA), sob Parecer n. ${ }^{\circ}$ 488.334, de 27 de setembro de 2013.

\section{RESULTADOS}

O estudo analisou 96 prontuários de crianças atendidas em um serviço de referência em atenção às pessoas com estomia localizado na cidade de Belém, estado do Pará, Brasil.

Os dados sociodemográficos da Tabela 1 evidenciaram o predomínio do sexo masculino - 58,33\% ( $\mathrm{n}=56)-$, da faixa etária entre 0 e 3 anos - 48,96\% ( $n=47)$-, da categoria sem escolaridade $-48,95 \%(n=47)-$, de procedentes de outros municípios do estado do Pará - 52,08\% $(\mathrm{n}=50)$ - e de renda familiar mensal entre um e três salários-mínimos - 40,63\% ( $\mathrm{n}=39$ ).

No Gráfico 1 , verificamos que a causa básica geradora de estomia de maior percentual foi a malformação congênita 58,32\% ( $\mathrm{n}=56)$ - , seguida por outras causas - 18,75\% $(\mathrm{n}=18)$. Com 7,03\% ( $\mathrm{n}=7)$, vieram os traumas. A neoplasia contabilizou 3,13\% (n=3), e em 3,13\% (n=3) das fichas não havia essa informação.

Tabela 1. Distribuição de crianças com estomia atendidas no serviço de referência no estado do Pará, Brasil, segundo as variáveis sociodemográficas, Belém, Pará, Brasil, 2013.

\begin{tabular}{|c|c|c|}
\hline Variáveis & $\mathrm{n}$ & $\%$ \\
\hline \multicolumn{3}{|l|}{ Sexo } \\
\hline Masculino & 56 & 58,33 \\
\hline Feminino & 40 & 41,67 \\
\hline \multicolumn{3}{|l|}{ Faixa etária (anos) } \\
\hline $0-3$ & 47 & 48,96 \\
\hline $4-6$ & 12 & 12,5 \\
\hline $7-9$ & 17 & 17,71 \\
\hline $10-12$ & 20 & 20,83 \\
\hline \multicolumn{3}{|l|}{ Escolaridade } \\
\hline $\begin{array}{l}\text { Sem escolaridade, pois não } \\
\text { estão em idade escolar }\end{array}$ & 47 & 48,95 \\
\hline $\begin{array}{l}\text { Sem escolaridade, porém } \\
\text { em idade escolar }\end{array}$ & 11 & 11,46 \\
\hline $\begin{array}{l}\text { Ensino fundamental } \\
\text { incompleto }\end{array}$ & 35 & 36,46 \\
\hline Sem informação & 3 & 3,13 \\
\hline \multicolumn{3}{|l|}{ Procedência } \\
\hline $\begin{array}{l}\text { Demais municípios } \\
\text { do estado do Pará }\end{array}$ & 50 & 52,08 \\
\hline Belém & 46 & 47,92 \\
\hline \multicolumn{3}{|l|}{ Renda familiar (salário-mínimo) } \\
\hline Sem renda fixa & 32 & 33,34 \\
\hline $1-3$ & 39 & 40,63 \\
\hline $3-5$ & 3 & 3,12 \\
\hline$\geq 5$ & 1 & 1,04 \\
\hline Sem informação & 21 & 21,87 \\
\hline Total & 96 & 100,00 \\
\hline
\end{tabular}


Em relação ao tipo de estomia, houve predomínio da colostomia - 60,43\% ( $\mathrm{n}=58)$ - e da ileostomia - 29,16\% $(\mathrm{n}=28)$ (Tabela 2).

Os dados clínicos da Tabela 3 revelaram o predomínio das estomias de caráter temporário - 83,33\% $(n=80)$; no quadrante inferior esquerdo $-46,87 \%$ ( $\mathrm{n}=45)$; com modo de exteriorização de duas bocas próximas - 31,26\% $(n=30)$ ou terminal $-31,26 \%$ ( $n=30)$; e diâmetro de estomia entre 20 e $29 \mathrm{~mm}-$ $36,45 \%(n=35)$. Não foram evidenciadas complicações na pele periestoma em 59,38\% ( $\mathrm{n}=57)$ das crianças, porém 38,54\% $(\mathrm{n}=37)$ delas desenvolveram dermatite. Não foram observadas complicações na estomia em $82,31 \%(\mathrm{n}=79)$ das crianças.

Gráfico 1. Percentual de crianças com estomia atendidas no serviço de referência no estado do Pará, Brasil, segundo a causa básica da estomia, Belém, Pará, Brasil, 2013.

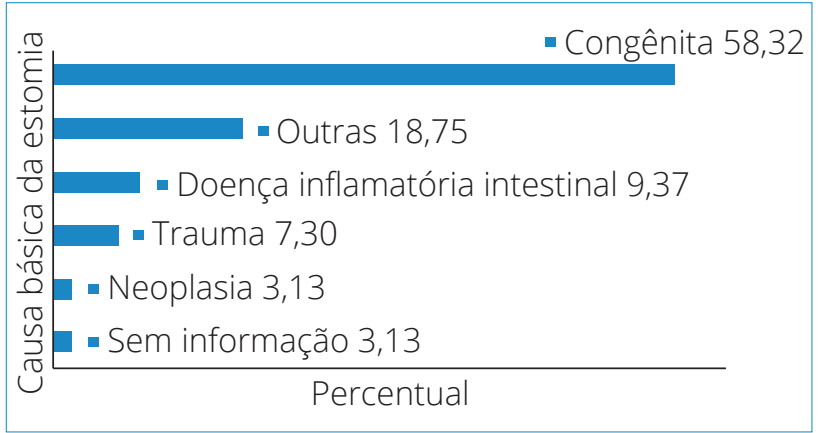

Tabela 2. Distribuição de crianças com estomia atendidas no serviço de referência no estado do Pará, Brasil, segundo o tipo de estomia, Belém, Pará, Brasil, 2013.

\begin{tabular}{lcc}
\hline Tipo de estomia & $\mathbf{n}$ & $\%$ \\
\hline Ileostomia & 28 & 29,16 \\
\hline Colostomia cólon descendente & 19 & 19,80 \\
\hline Colostomia cólon transverso & 15 & 15,63 \\
\hline Colostomia cólon ascendente & 13 & 13,54 \\
\hline Colostomia sigmoide & 11 & 11,46 \\
\hline Nefrostomia & 2 & 2,08 \\
\hline Vesicostomia & 1 & 1,04 \\
\hline Gastrostomia & 1 & 1,04 \\
\hline Sem informação & 6 & 6,25 \\
\hline Total & 96 & 100,00 \\
\hline
\end{tabular}

Tabela 3. Distribuição de crianças com estomia atendidas no serviço de referência no estado do Pará, Brasil, segundo caráter, localização, modo de exteriorização, diâmetro, complicações na pele periestoma e estomia, Belém, Pará, Brasil, 2013.

\begin{tabular}{|c|c|c|}
\hline Variáveis & $\mathrm{n}$ & $\%$ \\
\hline \multicolumn{3}{|l|}{ Caráter da estomia } \\
\hline Temporário & 80 & 83,33 \\
\hline Definitivo & 11 & 11,46 \\
\hline Sem informação & 5 & 5,21 \\
\hline \multicolumn{3}{|l|}{ Localização da estomia } \\
\hline Quadrante inferior esquerdo & 45 & 46,87 \\
\hline Quadrante inferior direito & 21 & 21,88 \\
\hline Quadrante superior esquerdo & 18 & 18,75 \\
\hline Quadrante superior direito & 7 & 7,29 \\
\hline Sem informação & 3 & 3,13 \\
\hline Outros & 2 & 2,08 \\
\hline \multicolumn{3}{|l|}{ Modo de exteriorização } \\
\hline Duas bocas próximas & 30 & 31,26 \\
\hline Terminal & 30 & 31,26 \\
\hline Em alça & 24 & 25,00 \\
\hline Duas bocas distantes & 7 & 7,28 \\
\hline Sem informação & 5 & 5,20 \\
\hline \multicolumn{3}{|l|}{ Diâmetro da estomia (mm) } \\
\hline $10-19$ & 14 & 14,58 \\
\hline $20-29$ & 35 & 36,45 \\
\hline $30-39$ & 27 & 28,12 \\
\hline $40-49$ & 10 & 10,42 \\
\hline $50-59$ & 7 & 7,30 \\
\hline Sem informação & 3 & 3,13 \\
\hline \multicolumn{3}{|l|}{ Complicações na pele periestoma } \\
\hline Sem complicação & 57 & 59,38 \\
\hline Dermatite & 37 & 38,54 \\
\hline Lesões pseudoverrugosas & 1 & 1,04 \\
\hline Candidíase & 1 & 1,04 \\
\hline \multicolumn{3}{|l|}{ Complicações na estomia } \\
\hline Sem complicação & 79 & 82,31 \\
\hline Prolapso & 7 & 7,29 \\
\hline Hérnia & 4 & 4,16 \\
\hline Retração & 4 & 4,16 \\
\hline Outras & 2 & 2,08 \\
\hline Total & 96 & 100,00 \\
\hline
\end{tabular}




\section{DISCUSSÃO}

Os resultados deste estudo apresentam informações relevantes quanto às características sociodemográficas e clínicas de crianças com estomia com base na análise de 96 prontuários. Constatamos o maior percentual de crianças com estomia do sexo masculino, com idade entre 0 e 3 anos e sem escolaridade.

Em estudo realizado no Programa de Cuidado Integral à Criança e ao Adolescente Estomizado e sua Família, da Faculdade de Enfermagem da Universidade Nacional da Colômbia, em 2004, os resultados evidenciaram que 67\% $(n=55)$ das crianças entrevistadas era do sexo masculino, e a variável escolaridade não se aplicou em 49 delas, por se tratar de lactentes ${ }^{9}$. Os motivos que impediam as crianças de frequentar a escola estavam relacionados a problemas de saúde, à distância entre a escola e sua residência, ao temor dos pais/responsáveis de expor a criança ao desprezo ou ainda à possibilidade de acidentes referentes à estomia fora do lar - infiltrações, rupturas ou desprendimento da bolsa, entre outros 9 . A maioria das crianças (69\%) era procedente de localidades distantes do distrito da capital ${ }^{9}$.

Outro estudo realizado em um ambulatório de estomaterapia de um hospital público do Distrito Federal (DF), Brasil, em 2013, fundamentado em 50 prontuários de crianças e adolescentes com estomia, revelou que $62 \%$ era do sexo masculino. A maioria estava na faixa etária de $0 \mathrm{a}$ 5 anos (84\%), e 60\% era oriundo de áreas administrativas do Distrito Federal ${ }^{8}$.

A variável escolaridade foi investigada em um estudo?, e seus resultados são semelhantes aos resultados aqui apresentados. As escolas, da educação infantil e do ensino fundamental, devem oferecer programas de educação permanente para seus educadores que possam capacitá-los quanto à atenção e integralização das crianças com deficiência, nesse caso da criança com estomia. Esse processo é de extrema importância não somente para a socialização da criança e melhoria do processo de adaptação ante o processo de reabilitação, mas principalmente por proporcionar aos pais/ responsáveis segurança no que concerne à autonomia da criança para além de sua residência.

A maioria das crianças provinha de municípios distantes da capital do estado. Esse dado provavelmente justifica-se por se tratar do único serviço de referência especializado na atenção à pessoa com estomia do estado do Pará. Investigação realizada com crianças e adolescentes constatou que $60 \%(\mathrm{n}=30)$ residia em áreas administrativas do Distrito Federal, e os demais, 40\% ( $n=20)$, eram procedentes de outros estados, como Goiás e Maranhão ${ }^{8}$. Vale ressaltar que o Distrito Federal é uma unidade atípica da federação, não possui municípios, porém é dividido em regiões administrativas conhecidas como cidades-satélites, com restrita autonomia administrativa ${ }^{12}$.

A possibilidade de acessar serviços de saúde localizados o mais próximo possível da residência dos cidadãos foi determinada desde a promulgação da Norma Operacional da Assistência à Saúde (NOAS), a qual ampliou as responsabilidades dos municípios na atenção básica, definiu o processo de regionalização da assistência e criou mecanismos para o fortalecimento da capacidade de gestão do Sistema Único de Saúde (SUS) ${ }^{13}$.

A proximidade entre a residência e o serviço de saúde, além de facilitar o deslocamento do cidadão, diminui as despesas com transporte, alimentação e hospedagem, evitando riscos associados a outros agravos - tais como acidentes nas estradas - ou fluviais, quedas de idosos, desconforto físico e emocional ${ }^{14}$.

A renda familiar média mensal das crianças com estomia foi equivalente à do estudo realizado no hospital público de Teresina, (PI) com 19 participantes, que revelou que a maioria, 52,63\% $(\mathrm{n}=10)$, possuía renda familiar compreendida entre 2 e 3 salários-mínimos ${ }^{5}$. O conhecimento da variável renda é essencial aos estudos que tratam do perfil das pessoas com estomia. Essa população tem elevados encargos financeiros com deslocamento para o serviço de saúde, muitas vezes distantes de sua residência, transporte, alimentação, compra de medicamentos, entre outros, influenciando diretamente na renda familiar. Por outro lado, cabe aqui ressaltar a problemática associada à aquisição de equipamentos e adjuvantes de proteção e segurança para estomia, no serviço público e nos planos de saúde, que obrigam as pessoas com estomia a comprar esses insumos essenciais para o autocuidado, impactando na sua renda familiar.

Houve o predomínio de malformações congênitas entre as crianças como causa básica da estomia. De acordo com a literatura consultada, as malformações congênitas são prevalentes entre nascidos vivos. Em estudo realizado em São Luís (MA), Brasil, no período de 2002 a 2011, constatou-se que, do total de 180.298 nascidos vivos (NV) registrados no Sistema de Informações sobre Nascidos Vivos (Sinasc), 875 $(0,49 \%)$ apresentaram algum tipo de malformação congênita, e, destes, 55,2\% eram do sexo masculino ${ }^{15}$. 
Outro estudo ${ }^{16}$ realizado com 27.564 recém-nascidos selecionados aleatoriamente em grupos caso e controle com base nos NV, no município de São Paulo (SP), no período de 2007 a 2011, revelou a prevalência de anomalia congênita em 1,2\% dos NV e evidenciou que a frequência do sexo masculino foi maior tanto no grupo com anomalia congênita $(57,69 \%)$ quanto no sem anomalia $(50,9 \%)$.

A doença de Hirschsprung é uma malformação congênita do intestino grosso caracterizada pela ausência de células ganglionares parassimpáticas intrínsecas no plexo submucoso e mioentérico em distintas porções do intestino. Sua incidência é de cerca de 1:5.000 nascidos vivos e o problema acomete predominantemente o sexo masculino, na razão de $4: 1^{17}$.

Quanto ao tipo de estomia apresentado pelas crianças, a colostomia foi prevalente neste estudo. Esse dado vem ao encontro da investigação desenvolvida com essa população no $\mathrm{Brasil}^{8}$. Em pediatria a colostomia é o tipo mais comum entre as estomias intestinais, na maioria das vezes de caráter temporário ${ }^{18}$. A doença de Hirschsprung constitui uma indicação clássica de colostomia por obstrução do cólon em crianças, com exceção dos casos em que é possível realizar o tratamento cirúrgico da doença de base ${ }^{19}$. Esse dado consiste numa importante informação para o planejamento assistencial às crianças e aos seus pais/responsáveis e ainda na previsão de equipamentos e adjuvantes de proteção e segurança para estomia nos serviços de saúde público e privado.

Os recém-nascidos com malformação anorretal (imperfuração anal) têm indicação cirúrgica à colostomia para descompressão do trato intestinal ${ }^{19}$. Os resultados também apontaram um percentual significativo de crianças com ileostomia, estomia do segmento ileal do intestino delgado, com saída involuntária e contínua de efluente de cor amarelo-esverdeada, odor ácido ou inodoro e pH alcalino. $\mathrm{O}$ volume de efluente eliminado pela ileostomia em crianças é aproximadamente $1 \mathrm{~mL} / \mathrm{kg} / \mathrm{h}$, fato que demanda atenção especializada e interdisciplinar, por conta da predisposição da ocorrência de desequilíbrio hidroeletrolítico ${ }^{18}$.

A maioria das crianças possuía estomia de caráter temporário. Resultados semelhantes foram encontrados em outros estudos ${ }^{8,9}$. Por ser estomia temporária, é possível a reconstituição do trânsito intestinal (RTI), dependendo da causa geradora da estomia ${ }^{1}$. A partir do momento em que a criança tem indicação médica para a cirurgia de RTI, o enfermeiro estomaterapeuta pode colaborar efetivamente, esclarecendo aos pais/responsáveis sobre a documentação necessária para o agendamento da nova cirurgia, garantir o retorno ambulatorial durante a presença da estomia, orientar para a nova intervenção cirúrgica, acompanhando todo o processo até mesmo após a alta hospitalar.

A localização predominante da estomia nas crianças foi o quadrante inferior esquerdo do abdome, local em que geralmente é realizada colostomia descendente cujo efluente se caracteriza por fezes semiformadas a formadas ${ }^{18}$. A demarcação pré-operatória da estomia constitui um procedimento técnico importante de competência do enfermeiro estomaterapeuta, ou não, que visa favorecer um local adequado para a confecção da estomia, contribuindo para a diminuição de riscos e complicações, tais como hérnia paraestomal, prolapso, retração, descolamento mucocutâneo, entre outros ${ }^{20}$.

Quanto ao modo de exteriorização da estomia, prevaleceram o terminal e o de duas bocas próximas, cada um equivalendo a $31,26 \%(n=30)$. Um estudo feito no estado do Mato Grosso do Sul com análise de 178 prontuários evidenciou o modo de exteriorização da estomia do tipo terminal em $68,4 \%$ dos $\operatorname{casos}^{21}$. No caso de crianças com imperfuração anal, a conduta cirúrgica tem sido a realização de uma estomia com duas bocas, uma boca proximal funcionante e uma distal não funcionante ${ }^{19}$. O segmento intestinal a ser exteriorizado depende do local comprometido do intestino, do tipo de afecção, das condições clínicas do doente e da preferência do cirurgião ${ }^{22}$. A área do abdome é anatomicamente menor na criança, e em casos de estomia com modo de exteriorização de duas bocas distantes há dificuldade de adaptação da base adesiva, mesmo do tipo infantil, ocasionando desconforto para a criança e ansiedade dos pais no tocante ao processo de cuidar. Os pais devem receber orientações minuciosas do enfermeiro estomaterapeuta concernente ao manejo do medidor da estomia e dos diferentes tipos de bases adesivas disponibilizadas pelo SUS.

$\mathrm{O}$ diâmetro mais comum da estomia foi entre 20 e $29 \mathrm{~mm}$. Essa variável constitui um dos dados importantes ao gerenciamento dos serviços de saúde, podendo ser utilizada para realizar a previsão e provisão dos equipamentos e adjuvantes de segurança e proteção para estomia, necessários à demanda dos usuários ${ }^{23}$. Essa variável implica diretamente o corte adequado da base adesiva do equipamento coletor no abdome, evitando fuga do efluente e, consequentemente, prevenindo lesões na pele periestoma.

No que se refere às complicações na pele periestoma, a maioria das crianças não apresentou complicação, provavelmente por se tratar de crianças com colostomia, cujo efluente 
é caracterizado por fezes semiformadas a formadas, diminuindo o risco de prejuízo da integridade da pele ao redor da estomia. Entretanto, um percentual significativo de crianças deste estudo desenvolveu dermatite, provavelmente associada aos casos de ileostomia, cujo efluente contém enzimas digestivas de elevado teor de proteases e lípases e, em contato com a pele em curto período de tempo, provoca dermatite. Em pesquisa do Programa de Ostomizados do Centro de Especialidades Médicas da Prefeitura de Campo Grande (MS), a dermatite da pele periestoma foi evidenciada em $28,7 \%$ de 178 pacientes investigados ${ }^{21}$. Outro estudo documental retrospectivo que analisou 443 prontuários em Belém (PA) constatou que 82,39\% (n=365) dos casos apresentaram dermatite periestoma ${ }^{11}$. O comprometimento da integridade da pele periestoma pode ser resultante do contato direto do efluente com a pele e da sensibilidade aos componentes da base adesiva do equipamento coletor e dos adjuvantes ${ }^{1,24,25}$

Com relação às complicações na estomia, a maioria das crianças não teve nenhuma complicação. Os resultados deste estudo divergem dos estudos realizados em Campo Grande e Belém ${ }^{11,21}$,nos quais foi saliente a prevalência de complicações na estomia. Para diminuir o aparecimento das complicações, é fundamental a orientação do enfermeiro estomaterapeuta aos pais/responsáveis da criança no período pós-operatório mediato. $\mathrm{O}$ profissional deve enfatizar os sinais de complicações, como sangramento, mudança na coloração e protrusão da estomia, abaulamento no abdome, e a conduta a ser tomada na vigência de qualquer intercorrência.

Ao receber uma criança com estomia em um serviço de saúde público ou privado, o enfermeiro estomaterapeuta deve buscar estratégias para o seu cuidado integral em conjunto com os pais/responsáveis. Precisa realizar o acompanhamento do crescimento e do desenvolvimento, orientar acerca do retorno ao ambiente escolar de acordo com a faixa etária, do manejo com a estomia no domicílio e em outros ambientes, da realização de atividades de recreação/lazer peculiares à cultura regional, quanto ao banho de rio/igarapé, da ingestão de frutos da região, tais como açaí, manga, cupuaçu, bacuri, entre outros. Além disso, proceder à avaliação da estomia e pele periestoma, para garantir a prescrição de equipamentos coletores e adjuvantes de proteção e segurança, em conformidade com as características individuais. $\mathrm{O}$ conjunto desses procedimentos é essencial à reabilitação da criança com estomia.

\section{CONCLUSÃO}

A maioria das crianças atendidas no serviço de referência no estado do Pará, Brasil, é do sexo masculino, procedente dos demais municípios do Pará, na faixa etária de 0 a 3 anos, sem escolaridade - por não estarem em idade escolar —, com renda familiar média mensal entre um e três salários-mínimos. A causa básica da cirurgia geradora da estomia foi a malformação congênita, resultando na confecção de colostomia, terminal ou em duas bocas próximas, temporária, com diâmetro de 20 a $29 \mathrm{~mm}$, localizada no quadrante inferior esquerdo. A maioria das crianças não apresentou complicações na pele periestoma nem na estomia.

Ressalta-se que uma das limitações da pesquisa foi a falta de informações de dados nos prontuários das crianças pesquisadas referentes a algumas variáveis do estudo. Os resultados desta investigação podem subsidiar gestores e profissionais da saúde ao planejamento de ações em atenção à saúde das crianças com estomia.

\section{REFERÊNCIAS}

1. Santos VLCG, Cesaretti IUR. Assistência em estomaterapia: cuidando de pessoas com estomia. $2^{\text {a }}$ ed. São Paulo: Atheneu; 2015.

2. Brasil. Estatuto da Criança e do Adolescente (ECA). Lei n. ${ }^{\circ}$ 8. 069, de 13 de julho de 1990. $3^{\text {a }}$ ed. Brasília: Câmara dos Deputados/Coordenação de Publicações; 2001.

3. Brasil. Decreto n. ${ }^{\circ}$ 5.296, de 2 de dezembro de 2004 Regulamenta as leis n. ${ }^{\circ} 10.048$, de 8 de novembro de 2000 , que dá prioridade de atendimento às pessoas que especifica, e n. 10.098, de 19 de dezembro de 2000, que estabelece normas gerais e critérios básicos para a promoção da acessibilidade das pessoas portadoras de deficiência ou com mobilidade reduzida, e dá outras providências. Brasília, 2004.
4. Brasil. Presidência da República. Lei n. 13.146 , de 6 de julho de 2015. Institui a Lei Brasileira de Inclusão da Pessoa com Deficiência (Estatuto da Pessoa com Deficiência). Brasília, 6 jul. 2015. [acesso 20 nov 2015]. Disponível em: <http:// www.planalto.gov.br/ccivil_03/_Ato2015-2018/2015/Lei/ L13146.htm>

5. Luz MHBA, Andrade DS, Amaral HO, Bezerra SMG, Benício CDAV, Leal ACA. Caracterização dos pacientes submetidos a estomas intestinais em um hospital público de Teresina-PI. Texto Contexto Enferm. 2009;18(1):140-6.

6. Martins PAF, Alvim NAT. Perspectiva educativa do cuidado de enfermagem sobre a manutenção da estomia de eliminação. Rev Bras Enferm. 2010;64(2):322-7. 
7. Brasil. Ministério da Saúde. Portaria n. ${ }^{\circ} 793$, de 24 de abril de 2012. Institui a Rede de Cuidados à Pessoa com Deficiência no âmbito do Sistema Único de Saúde. Brasília, 2012.

8. Monteiro SNC, Kamada I, Silva AL, Souza TCR. Perfil de crianças e adolescentes estomizados atendidos de um hospital público do Distrito Federal. Estima. 2014;12(3):23-32.

9. Guerrero S, Tobos LS. Quem são os usuários do Programa Cuidado Integral à Criança e ao Adolescente Ostomizado. Avanços em Enfermagem. 2005;23(2):18-26.

10. Pereira SSS, Quito MV. NOAS: houve impacto para o SUS? Revista de Direito Sanitário. 2004;5(1):40-54.

11. Silva AC, Silva GNS, Cunha RR. Caracterização de pessoas estomizadas atendidas em consulta de enfermagem do serviço de estomaterapia do município de Belém-PA. Estima. 2012;10(1):12-9.

12. Estados Brasileiros, Distrito Federal. [acesso 20 nov. 2015] Disponível em: <http://www.portalbrasil.net/estados_df.htm>

13. Brasil. Ministério da Saúde. Regionalização da Assistência à Saúde: aprofundando a descentralização com equidade no acesso: Norma Operacional da Assistência à Saúde: NOASSUS 01/02 e Portaria MS/GM n. ${ }^{\circ}$ 373, de 27 de fevereiro de 2002, e regulamentação complementar. Brasília, 2002.

14. Pontes APM, Cesso RGD, Oliveira DC, Gomes AMT. Facilidades de acesso reveladas pelos usuários do Sistema Único de Saúde. Rev Bras Enferm. 2010;63(4):574-80.

15. Rodrigues LS, Lima RHS, Costa LC, Lucena RF. Características das crianças nascidas com malformações congênitas no município de São Luís, Maranhão, 2002-2011. Epidemiol Serv Saúde. 2014;23(2):295-304.

16. Mendes CQS, Avena MJ, Mandetta MA, Ballieiro MMFG. Prevalência de nascidos vivos com anomalias congênitas no município de São Paulo. Rev Soc Bras Enferm Ped. 2015;15(1):7-12.
17. Amiel J, Lyonnet S. Hirschprung disease, associated syndromes, and genetics: a review. J Med Genet. 2001;38(11):729-39.

18. Kayo CMM, Yamamoto MS, Pellegrino DMS, Del Cistia MEGF, Carvalho WAF. Cuidando de crianças com estomia. In: Santos VLCG, Cesaretti IUR. Assistência em estomaterapia: cuidando de pessoas com estomia. $2^{a}$ ed. São Paulo: Atheneu; 2015. p. 205-42.

19. Rocha RFC, Velhote MCP. Estomias na criança. In: Santos VLCG, Cesaretti IUR. Assistência em estomaterapia: cuidando de pessoas com estomia. $2^{a}$ ed. São Paulo: Atheneu; 2015. p. 63-74.

20. Aguiar ESS, Santos AAR, Soares MJGO, Ancelmo MNS, Santos SR. Complicações do estoma e pele periestoma em pacientes com estomas intestinais. Estima. 2011:9(2):22-30.

21. Santos CHM, Bezerra MM, Bezerra FMM, Paraguassú BR. Perfil do paciente ostomizado e complicações relacionadas ao estoma. Rev Bras Coloproctol. 2007;27(1):16-9.

22. Rocha JJR. Estomas intestinais (ileostomias e colostomias) e anastomoses intestinais. Medicina Ribeirão Preto. 2011;44(1):51-6.

23. Cunha RR, Backes VMS, Ferreira AB. Características sóciodemográficas e clínicas de pessoas estomizadas: revisão de literatura. Estima. 2013;11(2):29-35.

24. Paula MA, Paula PR, Cesarretti IUR. Estomaterapia em foco e cuidado especializado. São Caetano do Sul: Yendis, 2014.

25. Poletto D, Gonçalves MI, Barros MTT, Anders JC, Martins ML. A criança com estoma intestinal e sua família: implicações para o cuidado de enfermagem. Texto Contexto Enferm. 2011;20(2):31-9. 